\title{
Asymmetry cascade multilevel modulation strategy research and its simulation
}

\author{
FENG Juan, LI Zi-peng
}

Institute of Engineering, Sichuan Normal University, Chengdu 610101, Sichuan

28014436@qq.com

Keywords: asymmetric; multilevel; inverter

\begin{abstract}
This paper proposes asymmetric cascaded multilevel inverter with unequal dc-link voltage ratio. Meanwhile, it's proved when asymmetric topology with dc-link ratio of $1: 2: 4$, the output voltage level can be maximized with the same number of devices. For this kind of topology, this paper designs the corresponding modulation strategy and current regulator. And for the structural features of asymmetric topology, the asymmetric cascaded multilevel inverter is simulated in the Matlab/Simulink.
\end{abstract}

\section{Introduction}

Multilevel inverter, as for the preferred choice of the high-power power electronic converter has got more and more attention in industry and academia. Now it has entered the industrial field successfully, therefore the multilevel inverter is regarded as a mature technology. This paper analysis the type of the cascade multilevel inverter; makes a comparison with the output level of multilevel topologies of different DC bus voltage; especially studies the bus voltage ratio of 1:2:4, according to its switching state and modulation method. It introduces the method of cascade multilevel modulation, and puts forward the corresponding modulation strategy for the asymmetric cascaded multilevel inverter. It also puts forward the hybrid frequency carrier modulation strategy considering the current intrusion problem. Meanwhile, this paper analyzes the control of this modulation method and implementation that joins the MPPT algorithm to study the topology of closed loop control strategy and the current regulator, and makes the simulation.

\section{The principle of asymmetry cascade multilevel topology}

People improved the CHB topology structure in order to increase the number of output voltage level, reduce the harmonic content and improve the waveform quality. The output level can be regarded as the multiple modules in series combination of each level due to the modular structure of $\mathrm{CHB}$ in series. For example, 5L- CHB, composed of two modules. When each module of the dc bus voltage Vdc, each unit of output level for Vdc, 0 , - Vdc. It can be seen from table 1, there are only five output levels in total $(2 \mathrm{Vdc}, \mathrm{Vdc}, 0,-\mathrm{Vdc}, 2 \mathrm{Vdc})$. The main reason is that both of them have the same output of a single cell voltage combination. As it is shown in the table 1 , for the total output level for certain (Vdc, $0,-\mathrm{Vdc})$, there are a lot of redundant state switches.

Table 1 state and output relationship of 5L-CHB inverter switches

\begin{tabular}{|c|c|c|}
\hline Total output level & First unit & Second unit \\
\hline $2 \mathrm{Vdc}$ & Vdc & Vdc \\
\hline \multirow[b]{2}{*}{$\mathrm{Vdc}$} & Vdc & 0 \\
\hline & 0 & $\mathrm{Vdc}$ \\
\hline \multirow{3}{*}{0} & $\mathrm{Vdc}$ & $-\mathrm{Vdc}$ \\
\hline & 0 & 0 \\
\hline & $-\mathrm{Vdc}$ & $\mathrm{Vdc}$ \\
\hline \multirow[t]{2}{*}{$-V d c$} & $-\mathrm{Vdc}$ & 0 \\
\hline & 0 & $-\mathrm{Vdc}$ \\
\hline$-2 \mathrm{Vdc}$ & $-\mathrm{Vdc}$ & $-\mathrm{Vdc}$ \\
\hline
\end{tabular}


In order to make full use of the device, and reduce the redundant state, the voltage of the DC side of the module unit is considered to be unequal and allocated according to a certain ratio. That can reduce the repetition of the synthesized voltage, so that can increase the number of output levels without increasing the number of units. The typical ratio is the II type, the ratio of the DC bus voltage of each unit is $1: 2: 4 \ldots \ldots$; the output levels can be obtained. Or III type, that is, the DC bus voltage ratio of each unit is 1:3:9.....; so, you can get output levels .In addition, you can also use II and III combination structure, namely 1:2:6 the mix of incremental topology. In order to achieve full PWM modulation, each of the cascade multilevel converter output voltage level for PWM modulation respectively is needed, thus realize fully PWM control on output voltage level totally.

Table 2 the comparison of asymmetrical Cascaded multilevel converter with Common ratio of DC bus voltage

\begin{tabular}{|l|l|l|l|}
\hline Cascaded H Bridge & DC bus ratio & Output voltage level & $\begin{array}{l}\text { PWM } \\
\text { modulation } \\
\text { degree }\end{array}$ \\
\hline 1 & $1 \mathrm{E}$ & 3 & Completely \\
\hline 2 & $1 \mathrm{E} 1 \mathrm{E}$ & 5 & Completely \\
\cline { 2 - 4 } & $1 \mathrm{E} 2 \mathrm{E}$ & 7 & Completely \\
\cline { 2 - 5 } & $1 \mathrm{E} 3 \mathrm{E}$ & 9 & part \\
\hline 3 & $1 \mathrm{E} 1 \mathrm{E} 1 \mathrm{E}$ & 7 & Completely \\
\cline { 2 - 5 } & $1 \mathrm{E} 2 \mathrm{E} 4 \mathrm{E}$ & 15 & Completely \\
\cline { 2 - 5 } & $1 \mathrm{E} 3 \mathrm{E} 9 \mathrm{E}$ & 27 & Completely \\
\cline { 2 - 4 } & $1 \mathrm{E} 2 \mathrm{E} 6 \mathrm{E}$ & 19 & Cort \\
\hline
\end{tabular}

The table 2 shows that under the same complex modulation, it can both achieve full PWM modulation, also achieve the high voltage and high level for the output of the dc bus voltage ratio of $1: 2: 6$. Because of its only switch state, the ratio of topology has the low voltage current reversing problem, which makes the stability of the system reduced. Comparatively speaking, that proximately 1:2:4 as this topology can be through the use of redundant switch state to solve the problems of the current flow backward, but the corresponding modulation will increase its difficulty. This paper emphasized the analysis shows proximately 1:2:4 as the modulation method of topology.

\section{Asymmetry cascade multilevel inverter}

The traditional PWM modulation the currents maybe flow backward, so this article uses the mixed frequency carrier modulation principle. For example, using the new hybrid frequency modulation method is shown in the table 3 and figure 1, the two are using the redundancy switch composite state. When the phase voltage is $\mathrm{N}$, the use of $\mathrm{N}-1$ same amplitude but different frequency modulation respectively with triangular carrier wave modulation paragraphs of can solve the problem of the reverse current. That method can solve the problems that the current flow backward. $4 \mathrm{u}$ unit adopts the triangle wave whose frequency is fc1 to modulation, $2 \mathrm{u}$ unit adopts the triangle wave, which frequency is fc2 to modulation; $1 \mathrm{u}$ unit adopts the triangle wave, which frequency is fc3 for modulation. The carrier frequency need to meet fc3 $>\mathrm{fc} 2>\mathrm{fc} 1$. 
Table 3 cascaded 1:2:4 Mixed Frequency carrier modulation principle

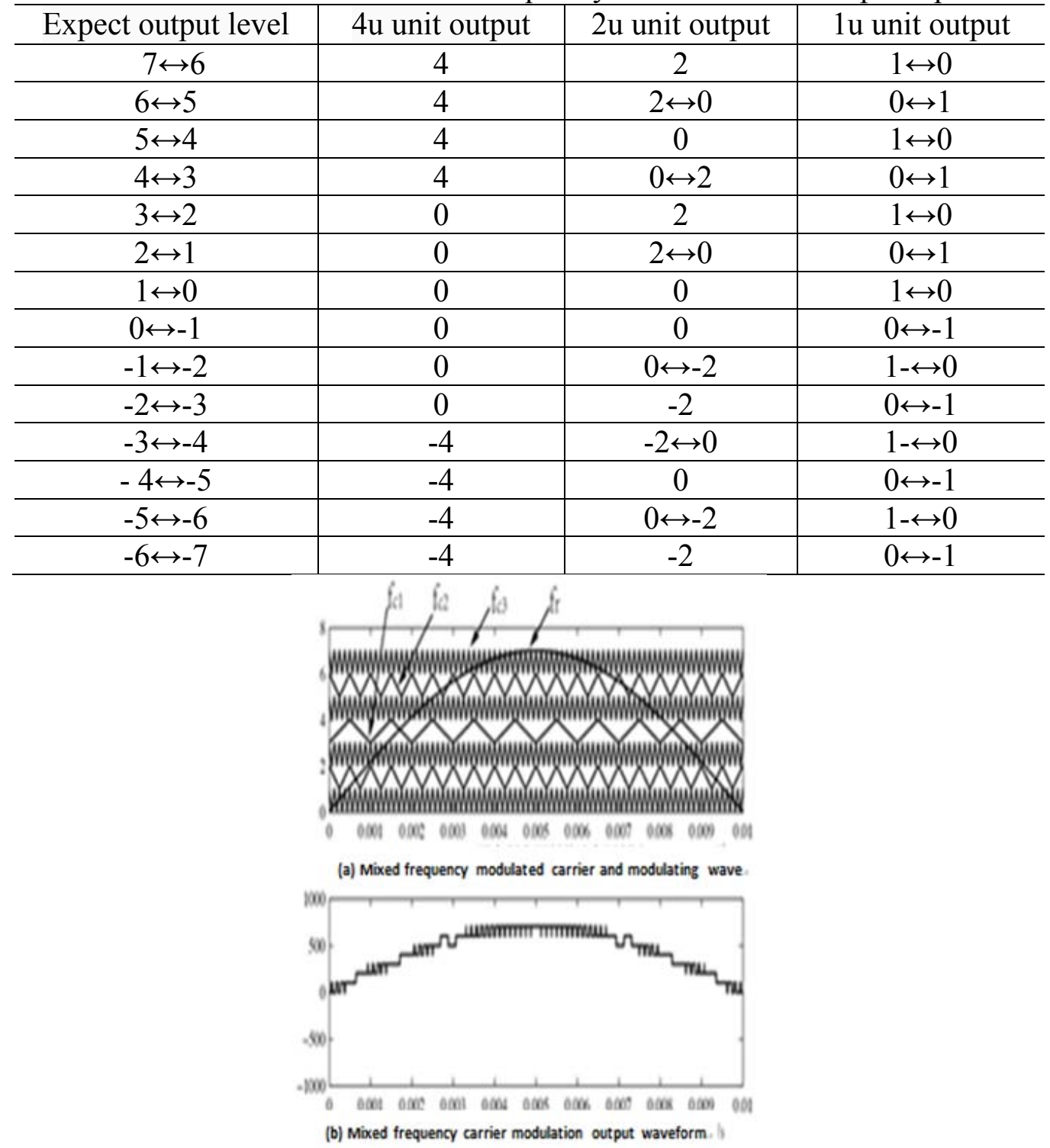

Figure 1 mixed frequency Carrier modulation waveform

The key point is that the $2 \mathrm{u}$ unit adopts the lower frequency triangular wave, which is in the second and the sixth layer shown in the figure 1 (a) .it makes it possible to PWM modulation at the above range, and makes that the selection of $\mathrm{f}_{-} \mathrm{c} 2$ is restricted by the high voltage IGCT switching frequency. That makes that the $6 \leftrightarrow 5 、 4 \leftrightarrow 3 、 2 \leftrightarrow 1$ and $-1 \leftrightarrow-2 、-3 \leftrightarrow-4 、-5 \leftrightarrow-6$ level output is alternately produced by the $1 \mathrm{u}$ unit IGCT and the $2 \mathrm{u}$ unit IGBT. That avoids the reverse current.

As shown in the table 3 and the figure 2 , only considering the positive half cycle parts, the $4 \mathrm{u}$ unit is only modulated in the $4 \leftrightarrow 3$ parts. But, because the $2 u$ unit need to modulate in the parts of $6 \leftrightarrow 5 、 4 \leftrightarrow 3 、 2 \leftrightarrow 1$, therefore the driving signal of $2 u$ unit is the superposition of the modulation signal which is shown in figure2(b) and figure2(a). But, the part of the judgment rules about high and low voltage is the opposite. Unit $1 \mathrm{u}$ need modulated in all parts. Therefore the driving signal of $1 \mathrm{u}$ is the combination of the modulated signal alone generated in figure 2(c) and the superposition of the modulated signal shown in figure 2(a) and figure 2(b). From the table 3, it is on the contrary that the judgment rule of $1 \mathrm{u}$ and $1 \mathrm{u} 2 \mathrm{u}$ about high and low voltage, every unit waveform and all the output waveform as shown in the figure3. From the figure 3, this scheme makes the output level of each unit consistent, and still uses the PWM modulation; it keeps the good quality of output voltage waveform, and solves the reverse current. As shown in figure 4.From analysis the traditional modulation scheme and the mixed frequency carrier modulation scheme, it can be seen that the 
mixed frequency modulation scheme has low THD, it improves the quality of output voltage waveform, but due to the low frequency carrier was introduced, the corresponding low harmonic content is increasing. This is the problem that should be considered when we design the frequency converter and output filter.
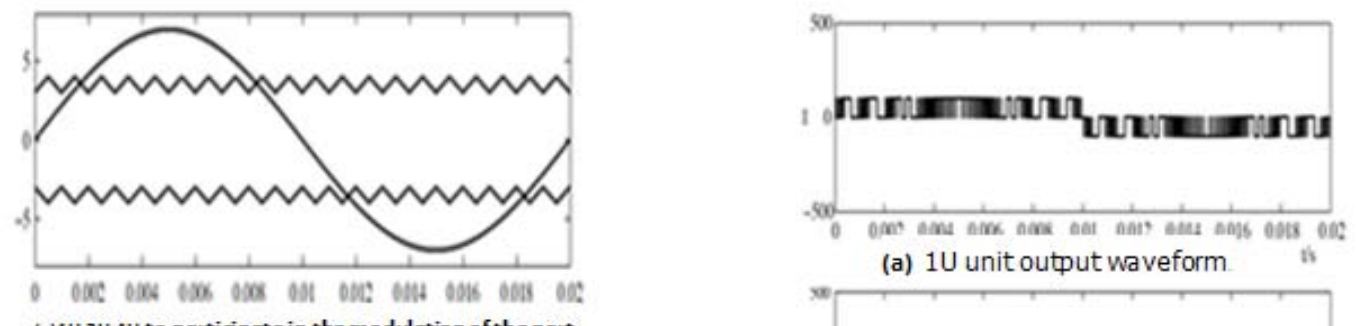

(a) $1 \mathrm{U}$ unit output waveform

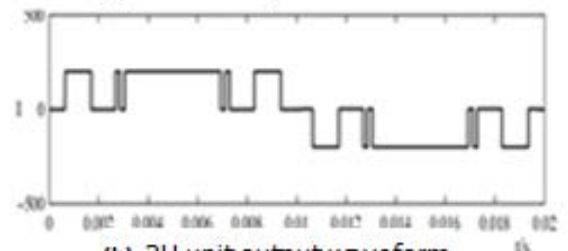

(b) $2 \mathrm{U}$ unit output waveform

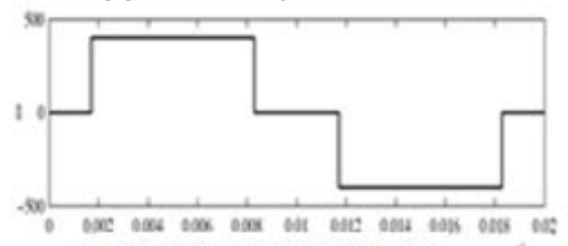

(c) $4 U$ unit output waveform.

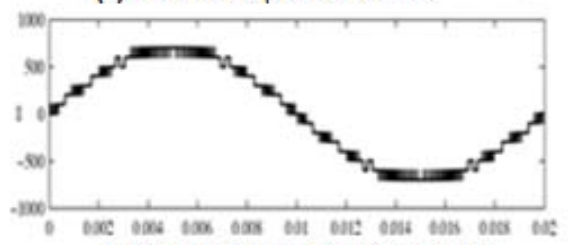

(d) Inverter output voltage wave

Figure 2 mixed frequency

Modulation waveform carrier units

Figure 3 mixed frequency carrier units and total output waveform

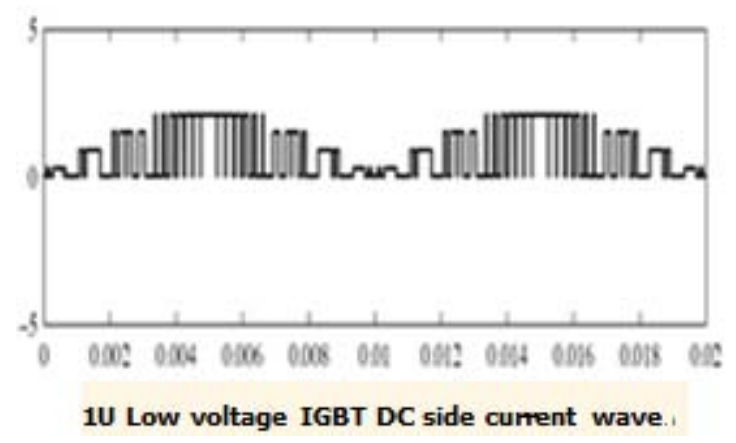

Figure 4 resistance load of low voltage de side IGBT unit current waveform

\section{Conclusions}

Through the above analysis of the principle of modulation, a modulation strategy is proposed for the asymmetric cascaded multilevel topology based on the DC bus voltage ratio of 1:2:4 in this article. As for the reverse current problems of appearing in the ratio of 1:2:4 topologic, this article uses the switching state redundancy and the hybrid frequency carrier modulation strategy to solve the problem. The system model is established in the Matlab, the whole modulation level is achieved, at the same time; the model is debugged in the experimental circuit. At last, the result shows that the reverse current problem has been effectively suppressed. 


\section{Author's brief introduction}

Feng Juan (1982), female, Chengdu, Sichuan province, lecturer, research direction is the electric drive and control,

E-Mail:28014436@qq.com; Tel: 13880710519

Address: Longquanyi district of Chengdu in Sichuan province Longcheng road normal university modern garden Zip code: 610101

\section{References}

[1] Kouro S, Malinowski M, Gopakumar K, et al. Recent advances and industrial applications of multilevel converters[J]. Industrial Electronics, IEEE Transactions on, 2010, 57(8): 2553-2580.

[2] Kouro S, Bernal R, Miranda H, et al. Direct torque control with reduced switching losses for asymmetric multilevel inverter fed induction motor drives[C]//Industry Applications Conference, 2006. 41st IAS Annual Meeting. Conference Record of the 2006 IEEE. IEEE, 2006, 5: 2441-2446.

3] wilsun xu.a, novak s. Power electronic technology [M]. Beijing: mechanical industry publishing house, 2006

[4] He Daqing Cai Xu. Modular multilevel converter limiter control and hybrid modulation [J]. Electric power automation equipment, 2012, 32 (4) : 63-66.

[5] would, Guo Dianlin guo-min Chen, jin-bo dong. Four quadrant operation of multilevel inverter control strategy research [J]. Power electronics technology, 2012, 46-48 (5) : 12-13 + 38.

[6] Wang Chen documentary, Yong-dong li. Multilevel converter topology relationship and a new topological [J]. Journal of electrotechnics, 2011,

$26(1): 92-92$. 